TITLE:

\title{
Optical Absorptions of Aliphatic Amino Acids in the Far Ultraviolet( Dissertation_全文)
}

\section{AUTHOR(S):}

Inagaki, Takashi

\section{CITATION:}

Inagaki, Takashi. Optical Absorptions of Aliphatic Amino Acids in the Far Ultraviolet. 京都 大学, 1973, 理学博士

ISSUE DATE:

1973-07-23

URL:

https://doi.org/10.14989/doctor.r2334

RIGHT: 
Optical Absorptions of Aliphatic Amino Acids in the Far Ultraviolet*

T. INAGAKI, Department of Physics, Osaka Kyoiku University, Ten-nojj, Osaka, Japan

Running Title: AIIPHATIC AMrNO ACIDS

* The experiment reported were performed at the Department of Physics, Kyoto University, Kyoto. 


\section{Synopsis}

The far ultraviolet absorptions of aliphatic anino acids such as glycine, alenine, eminobutyric ecid, valine and leucine were measured with their solid thin films down to $120 \mathrm{~nm}$. Four absorption bands arising from the carboxyl group were observed in the. 200-140 nm region. It was found that the band positions and intensities very systematically according to the number of carbon atoms in the aliphatic side chain. This result may be interpreted as a phenomenon similar to the hyper- and hypochromisms of aliphatic homopolypeptides in the far ultraviolet region. 


\section{INTRODUCTION}

Absorption spectra of amino acids in the near ultraviolet region have been extensively studied in the solution state ${ }^{1}$ and heve been very useful for optical studies of polypeptides. Most of the absoxptions in this region arise from the chromophoric side chains, and the absorptions due to the carboxyl and amino groups in amino acids hardly occur in this region. In the solution spectra of nonchromophoric amino acids such as aliphatic amino acids, in fact, we can find only absorption edges sterting from about $190 \mathrm{~nm}$, that is the usual short wavelength limit of solution study. Among these absorption edges, only slight variations which reflect the difference of the side chains can be seen. Therefore, it is important to obtain the spectra of nonchromophoric amino acids in the farther ultraviolet region and find out the effects of the side chain groups on them. In the spectral region below $190 \mathrm{~nm}$, however, samples in the solution state are no Ionger usuable because of the opacity of most solvents. Earlier Preiss and Setlow have carried out absorption measurements on glycine and alanine with their solid films down to $145 \mathrm{~nm}$ and obtained spectra considerably different from one another. Recently, Vinogrador and Dodonova ${ }^{3}$ have measured a number of solid films of aliphatic amino acids, including those of the above compounds, down to $115 \mathrm{~nm}$. For 
all of the compounds they found a intense band at $120 \mathrm{~mm}$ and a shoulder in the vicinity of 160-nm. However, their date are too diffuse to discuss the difference of spectral feature between compounds. Furthermore, they have not paid any attentions to the detailed structure of the spectral region called by them a "shoulder at $160 \mathrm{~nm}$ ".

In this paper well-resolved absorption spectra of solid films of aliphatic amino acids in $\alpha-\mathrm{L}-$ form $\left(\mathrm{NH}_{2}-\mathrm{CHR}-\mathrm{COOH}\right)$ measured in the region 280-120 nm are reported. Measurements have been carried out for glycine ( $R$ : H), alanine ( $R$ : $\mathrm{CH}_{3}$ ), aminobutyric acid ( $\mathrm{H}: \mathrm{C}_{2} \mathrm{H}_{5}$ ), valine ( $\mathrm{R}$ : $\mathrm{C}_{3} \mathrm{H}_{7}$ ) and leucine (R: $C_{4} B_{9}$ ). Isomers of the latter two compounds, norvaline, norleucine and isoleucine have also been measured. Of these compounds, aminobutyric acid, norvaline and norleucine have not been found in naturally occurring proteins. Analyzing the "shoulders at $160 \mathrm{~nm}$ ", we attempt to find out their detailed structures and the effects of the side chain (R) on them. Such a study may shed light on the optical properties of aliphatic homo-polypeptides in the far ultraviolet recently reported in the literatures. ${ }^{4-6}$ 


\section{EXPERIMENTAL}

For absorption measurements, a Shimadzu SGV-50 vacuum ultraviolet spectrophotometer has been employed. The details and equipments of this instrument have been previously described elsewhere. 7 A cold cathode hydrogen discharge lamp has been used as a radiation source. The emission from this lamp is a continuum in the region above $165 \mathrm{~nm}$ and a many-line spectrum in the region below $165 \mathrm{~nm}$. In the latter region, the intense 55 lines have been used for moasurements.

All compounds were measured at room temperature as solid thin films cast on LiF plates by vacuum sublimation. Sublimations were performed under pressure of about $1 \times 10^{-5}$ Torr and at temperatures as low as possible to avoid the decomposition of the compounds. It was ascertained from the following facts that the thermal decomposition has not occurred during the sublimation process. (1) All materials were deposited on a glass plate by racuum sublimation under the same condition as film preparations on Lif plates. These deposited materials dissolved in water, and their ultraviolet absorptions down to $195 \mathrm{~nm}$ were measured. These solutions gave the same spectre as the aqueous solutions of the original materials. (2) Preiss and Setlow ${ }^{2}$ made solid films of glycine and alanine by pipetting their aqueous solutions onto $\mathrm{CaF}_{2}$ substrates and drying slowly in a desiccator. This method 
does not include the possibility of decomposition at all. Our sublimed film spectra of the above compounds are in good agreement with their spectra, except for large light scattering losses of the latter spectra. Such a check was carried out also for the remaining compounds by preparing their solid films by the method of Preiss and Setlow. From these facts, the thermal decomposition may be concluded to be negligible in all of the compounds.

Sublimed films show non-negligible amounts of light scattering in absorption measurements. It was observed that the scattering losses from the films in the nonabsorbing region of $210 \mathrm{~nm}$ to $350 \mathrm{~nm}$ show power exponent dependences on wavelength from $\lambda^{-1}$ to $\lambda^{0}$ approximately. Then, the scattering optical density in absorbing region was estimated from data taken in the non-absorbing region assuming these observed wavelength dependences. 


\section{RESULTS AND ANALYSIS}

The results with light scattering corrections are shown in Figure 1. The figures are arranged in order of increasing number of carbon atoms in the side chain group from top to bottom. It can be seen that all amino acids studied exhibit, in addition to a continuous high background absorption, some structures in the low energy region which are considerably different from compound to compound. From glycine to norleucine the steep risings of intense background absorption and the first absorption maxima shift systematically to lower energies. The second apparent absorption maxima can be found in the spectra from norvaline to norloucine at near $160 \mathrm{~nm}$. Additional structure of a shoulder at near $180 \mathrm{~nm}$ is found for glycine, alanine, aminobutyric acid (hereafter abbreviated to $\triangle B A$ ) and isoleucine.

Detailed structures at low energy region were examined by reducing the spectra into gaussian band components. One possible decomposition of the spectra is presented in Figure 2. This anelysis wes performed as follow. At first the slopes of steep rising at $130-150 \mathrm{~nm}$ were extrapolated into the lower energy region, and the strong background absorptions were reduced from the spectra. This procedure can be carried out decisively for the spectra other than alanine and leucine spectra which have not an apparent break of absorption curve in the 
above wavelength region. For these two spectra the slopes of background absorption toward low energy region were determined by assuming that they do not largely differ from those of their adjacent spectra. Then, the resultant absorptions at low energy were decomposed into the definite number of gausian band components suggested by the humps which appear in the resultant absorption curves.

Spectral features of the present set of spectra arising from such reductions are typified by that of alanine. We can find four absorption bands in the region above $140 \mathrm{~nm}$ of alanine spectrum. In ordex to facilitate discussion, we have labelod the four bands in order as I, II, III and IV from low energy region. In the observed curve of alanine, the I-band corresponds to a extremely weak band at about 200 nm and the II-band corresponds to a small shoulder at near $180 \mathrm{~nm}$. The III-band is obviously the main band which has the absorption maximum located at $167 \mathrm{~nm}$ and the IV-band corresponds to a plateal between $160-140 \mathrm{~nm}$. There are good correlations between the absorption bend of alanine. and those of other.compounds shown below it. The I-band cannot be observed in all of the latter spectra, but the III- and IV-bands appear in all of them. The II-band can be found in the spectra of $A B A$ and isoleucine, though it is very weak in these compounds. From alanine to norleucine 
the II-band haxdly shifts, but the III- and IV-bands shift Iargely to the red. Next, les us consider the intensities, of the II- and IV-bands relative to that of the III-band for each compound. Absorption intensities can be obtained from the axeas below the band curives. It is found that with increasing number of carbon atoms in the side chain the absorption intensity relative to that of the III-band decreases for the II-band and increases for the IV-band.

If such correspondences of absorption bands among the compounds are accepted, the spectrum of glycine, whose absorption curve is considerably different from other ones, is readily interpreted. As there is no carbon atom in the side chain of this compound, it is expected from the trends of shifts of band positions and intensities that the III- and IV-bends of glycine are located at the shorter wavelength side as compared with the corresponding bands of alenine and that the absorption intensity relative to that of the IIIband is larger for the II-band and smaller for the IV-band than the corresponding one of alanine. Therefore, it is reasonable that for glyoine spectrum a weak band at $180 \mathrm{~nm}$ is identified as the II-band, a intense band at $158 \mathrm{~nm}$ as the III-band and a weak band at $145 \mathrm{~nm}$ as the IV-band. In addition to these bands, glycine shows a exceedingly weak absorption at ebout $200 \mathrm{~nm}$ corresponding to the I-band. 
Fig. 3

[able $]$.

In Pigure 3 the positions of band peaks are plotted with respect to the number of carbon atoms in the side chain. Systematic red shifts of band positions with increasing number of carbon atoms can be seen. It is to be noted that the separations between the III- and IV-bands are approximately constant and that these bands show considerably larger red shifts as compared with the II-band, though the magnitudes of them decrease with increase of the number of carbon atoms.

The ratios of intensity of the I-, II- and IV-bands to that of the III-band are summerized in Table I. Systematic variations according to the number of carbon atoms in the side chain can be found also in this table. In particular, a drastic increase of the intensity of the IV-band relative to that of the III-band is to be noted. This is ascertained in the observed absorption curves shown in Figure 1 as appearances of the second absorption maxima in the vicinity of $160 \mathrm{~nm}$.

As is well known, a reduction of a spectrum into gaussian band components is not unique, and in the present case the band positions and intensities largely depend on the extrapolation of the strong background absorption at high energy and the choice of bandwidths. However, for most cases of our spectra the reduction of the background absorption can be carried out without large arbitrariness, and if the 
bumps which appear in the resultant absorption curves are assumed to correspond to each absorption band the resultant absorptions at low energy can be decomposed into the definite number of band components. It is probable, therefore, that the reductions of the spectra performed here just reflects the essential features of optical absorptions of aliphatic amino acids. It seems to strengthen the validity of the present decompositions that they produce remarkable trends in band positions and intensities. A glance over a set of spectra shown in Figure 1 may suggest another interpretations, for example, that the structures at low energy of each spectrum essentially consist of two strong bands at near $180 \mathrm{~nm}$ and $160 \mathrm{~nm}$. Such an interpretation has been, however, ruled out after careful examinations, because it was found out that the resultant absorptions of alanine and $A B A$ cannot be decomposed into two band components without large deformations of band shape, in whatever reasonable way the strong background absorptions may be reduced. 


\section{DISCUSSION}

Four absorption bands at low energy obtained here seem to arise erom the carboxyl group $(-\mathrm{COOH})$, beceuse the amino and aliphatic groups do not absorp in the region above $150 \mathrm{~nm} .^{8}$ The far ultraviolet absorptions of simple carboxyl compounds such as formic acid (HCOOH), acetic acid ( $\mathrm{CH}_{3} \mathrm{COOH}$ ) and trifluoroacetic acid ( $\mathrm{CF}_{3} \mathrm{COOH}$ ) have been measured in the ges phase by Nagakura et al., 9 Barnes and Simpson ${ }^{10}$ and Basch et al. 11 . The latter two authors have found that the carboxyls show four bands in the same region as of the present study. The first weak bend has been classified as the first $n-\pi^{*}$ transition and the third intense band as the first $\pi-\pi^{*}$ transition. These assignments seem to be acceptable for the I- and III-bands obtained here, respectively. The assignments of the second and fourth bands of moderate intensities, however, have remained somewhat ambiguous. Basch et al. 11 have shown the possibility of $n-\sigma^{*}, \pi-\sigma^{*}, n^{\prime}-\pi^{*}$ and Rydberg transitions for these bands by a SCF calculation on carboxyls taking account of all valence-shell electrons. Mainly because of the experimental fact that these bands disappear in the condensed phase, they have proposed assignment as Rydberg transitions for both of them. However, these assignments cannot be accepted for the II- and IV-bands, since our spectra have been taken with the samples in the 
solid phase. The second and fourth bands of condensed carboxyls have been resolved for the first time in the present work. Those of the condensed simple carboxyls seem to be merged into tho adjacent more intense absorptions. It is, however, difficult to determine whether or not the second and fourth bands of amino acids correspond to those of gaseous simple carboxyls in their natures, since there are strong intermolecular interactions in the solid sample, involving the formations of hydrogen-bond, and the carboxyl group in amino acid is more or less perturbed with the amino and side chain groups within its own molecule.

It is well known 10,11 that gaseous spectra of simple amides $\left(-\mathrm{CONH}_{2}\right)$ have features very similar to one of gaseous carboxyls, that is, gas oous amides also exhibit four absorptions in the region where four bands of carboxyls occur. This has been ascribed to that both of them have a negative cinarged allylic electronic structure, 1 . e., four th-electrons in three T-molecular orbitals. Therefore, it is expected that the spectra reported here have features analogous to that of simple amides in the condensed phase. The latter has been obtained by Basch et al. 11 with formamide $\left(\mathrm{HCONH}_{2}\right)$ and by Momii and Urry ${ }^{4}$ with $\mathrm{N}-$ methyl acetemide $\left(\mathrm{CH}_{3} \mathrm{CONHCH}_{3}\right)$. However, in theae spectra only single band which seems to 
correspond to the first $\pi-\pi^{*}$ transition has been found in. the 190-180 nm region. On the other hand, it has been observed by many authors ${ }^{4-6}$ that solid pilms of aliphatic homopolypeptides show two absorption bands at near 190 and $165 \mathrm{~nm}$. In so far as these low-lying absorptions concerned, these polymers can be regarded as assemblies of amide chromophores (-CONH-) weakly interacting with each other. As is expected, this spectral feature is very similar to that of amino acids obtained here. These two bands presumably correspond to the III- and IV-bands, respectively. The assignment of the 190 nm band as the first $\pi-\pi^{*}$ trangition is accepted by many authors, but, so far, the assignment of $165 \mathrm{~nm}$ band has not been made. The above-mentioned fact that a resolvable band to which this $165 \mathrm{~nm}$ band corresponds is not observed in amide-monomer spectra taken in the condensed phase makes its assignment difficult.

In respeot to the intensities of polypeptide absorptions, a very interesting observation has been reported, ${ }^{4-6}$ that is, the intensity of the $165 \mathrm{~nm}$ band relative to that of the $190 \mathrm{~nm}$ band drastically changes from one polymer to another polymer. Employing the method of band analysis similar to present one, Onari ${ }^{6}$ has estimated the ratio of absorption intensity of the $165 \mathrm{~nm}$ band to that of the $190 \mathrm{~nm}$ band $\left(f_{165} / f_{290}\right)$ to be 0.49 for polyglycine, 0.75 for poly-L- 
valine, 1.04 for poly-L-alanine and 2.29 for poly-L-1eucine. Such a behavior of absorption intensity reminds us of that: of the III- and IV-bands of the present study, though in the case of amino acids the intensity changes are accompanied with rather large shifts of band position, whereas in the case of polypeptides they are accompanied with only slight band shifts. As shown in Table I, the ratio of intensity of the IV-band to that of the III-band $\left(f_{I V} / p_{I I I}\right)$ varies from 0.13 to 1.20 according to the number of carbon atoms in the side chain. The order of numerical values of ${ }_{I V} / s_{I I I}$ is in good agreement with that of $f_{165} / f_{190}$, except for the inversion of alanine and valine. These facts strongly suggest the respective correspondences of the III- and IV-bands to the 190 and $165 \mathrm{~nm}$ bends. The intensity change of polypeptide absorptions has been interpreted as due to difference of their conformations, but not due to difference of their side chains. Peptide chromophores in polymer are spatially configurated according to their conformations, and the variation of interchromophore interaction results from variability of the conformation. Pysh ${ }^{12}$ has carried out a calculation of the absorption intensity of interacting peptide groups for various conformations and obtained a result accounting well for expeximental facts qualitatively.

The remarkable trends of shifts of band positions and 
intensities shown in Figure 3 and Table I may be intexpreted essentially in terms of intermolecular perturbation as well as the case of polypeptides. It is not likely that such large spectral shifts are produced by the intramolecular perturbation from the aliphatic side chain indirectly attached to the carboxyl group through a $\alpha$-carbon atom. In fact, molecular arrangements considerably different from one another can be found in the available data of crystalline structures of aliphatic amino acids. ${ }^{13}$ Therefore, it is conceivable that the spectral shifts observed here are arising from the same oxigin as that of the shifts of polypeptide absorptions. It is not reasonable, however, to neglect completely the intramolecular perturbations from the side chain group. In the case of polypeptide, for example, the difference of intensity ratio $f_{165} / f_{190}$ between poly-Lalanine and poly-L-leucine cannot be explained by their conformations alone, since it has been ascertained that both of them have nearly same conformations ( $\alpha$-helices) in the measured samples. ${ }^{6}$ It is probable that in order to explain it successfully we must explicitly take account of the . intramolecular side chain effects: If any such effects are responsible for the shifts of peptide absorptions in polymer, similar ones should be expected for those of carboxyl absorptions in amino acid, because their side chains are 
situated at equivalent positions with respect to their chromophores. At the present stage of investigation, it is difficult to estimate how much extent the intramolecular perturbations from the side chafn contribute to the observed spectral shifts. Further study similar to the present one with the sample in which each molecule is isolated may provide any important informetions to clarify this point.

\section{Acknowledgement}

The author wishes to thank members of Goken for continuous encouragement. He is also grateful to Dr. T. Yamada and members of L. Jishuken for many helpful discussions. 


\section{References}

1. D. B. Wetlaufer, Advan. Protein Chem., 17, 320 (1962).

2. J. V. Preiss and R. Setlow, J. Chem. Phys., 25, 138 (1956).

3. I. P. Vinogrador and N. Ya. Dodonora, Opt. Spectrosc, 42,14 (1971).

4. R. K. Momii and D. W. Ưrry, Macromolecules, 1, 372 (1968).

5. J. L. Bensing and E. S. Pysh, Chem. Phys. Letters, 4, 120 (1969).

6. S. Onari, J. Phys, Soc. Japan, 30, 811 (1971).

7. T. Inagaki, J. Chem. Phys., 57, 2526 (1972).

8. J. W. Raymonda and W. T. Simpson', J. Chem. Phys., 47, 430 (1967).

9. S. Nagakura, K. Kaya and H. Tsubomura, J. Mol. Spectrosc., 13. 1 (1964).

10. E. E. Barnes and W. T. Simpson, J. Chem. Phys., 39, 670 (1963).

11. H. Basch, M. B. Robin and N. A. Kueblex, J. Chem. Phys., 49,5007 (1968).

12. E. S. Pysh, J. Chem. Phys,, 52, 4723 (1970).

13. R. W. G. Wyckoff, "Crystal Structures", second editiọn, vol. V, chap. XIV-D, Interscience Publishers, New Iork, 1966. 
TABLE I

Absorption intensities of the $I_{-}, I I-$ and IV-bands

relative to that of the III-band.

\begin{tabular}{|c|c|c|c|c|}
\hline Side chain & Compound & $\mathbf{f}_{I} / \mathbf{f}_{I I I}$ & $\mathbf{f}_{\text {II }} / \mathbf{f}_{\text {III }}$ & $f_{\text {IV }} / \mathbf{f}_{\text {III }}$ \\
\hline$-\mathrm{H}$ & glycine & 0.01 & 0.19 & 0.13 \\
\hline$-\mathrm{CH}_{3}$ & -.. Elanine & 0.01 & 0.09 & 0.22 \\
\hline$-\mathrm{C}_{2} \mathrm{H}_{5}$ & $\begin{array}{l}\text { aminobutyric } \\
\text { acid }\end{array}$ & - & 0.04 & 0.40 \\
\hline$-\mathrm{C}_{3} \mathrm{H}_{7}$ & $\begin{array}{l}\text { valine } \\
\text { norvaline }\end{array}$ & $\begin{array}{l}- \\
-\end{array}$ & $\begin{array}{l}- \\
-\end{array}$ & $\begin{array}{l}0.52 \\
0.63\end{array}$ \\
\hline & $\therefore$ isoleucine & - & 0.02 & 1.20 \\
\hline$-\mathrm{C}_{4} \mathrm{H}_{9}$ & leucine & - & - & 0.78 \\
\hline & norleucine & - & - & 0.87 \\
\hline
\end{tabular}




\section{FIGURE LEGEND}

Fig. 1. Absorption spectra of solid films of aliphatic $\alpha-L-a m i n o$ acids .

Pig.. 2. Decompositions of absorption spectra of aliphatic $\alpha$-L-amino acids into gaussian band components . (-) decomposed band component, (--) background absorption, ( $(\cdots)$ observed absorption curve.

Fig. 3. Positions of band peaks of aliphatic $\alpha$-L-amino acids ( $\mathrm{NH}_{2}-\mathrm{CHR}-\mathrm{COOH}$ ) plotted with respect to the number of carbon atoms in their side chains $(R)$. 


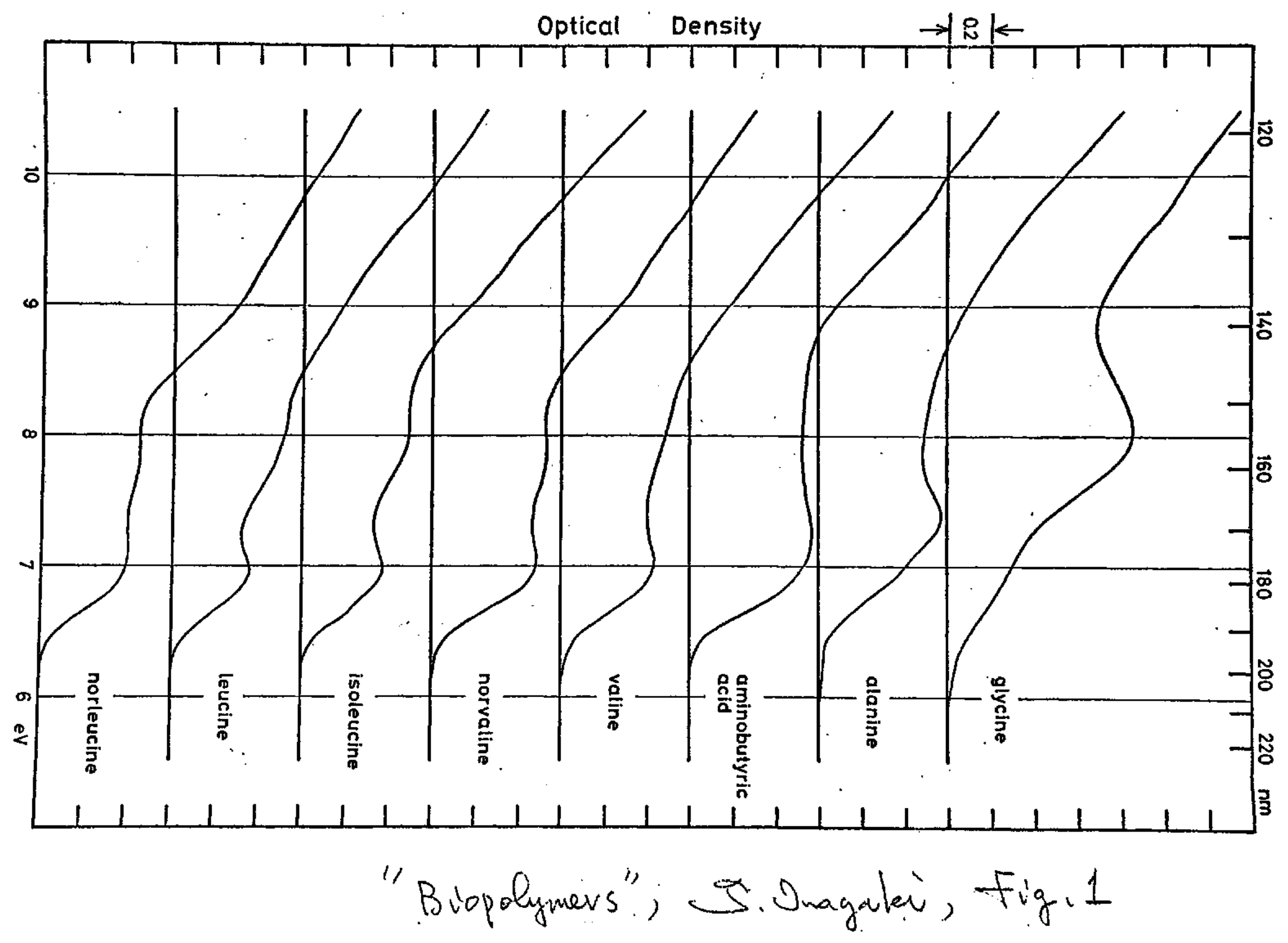




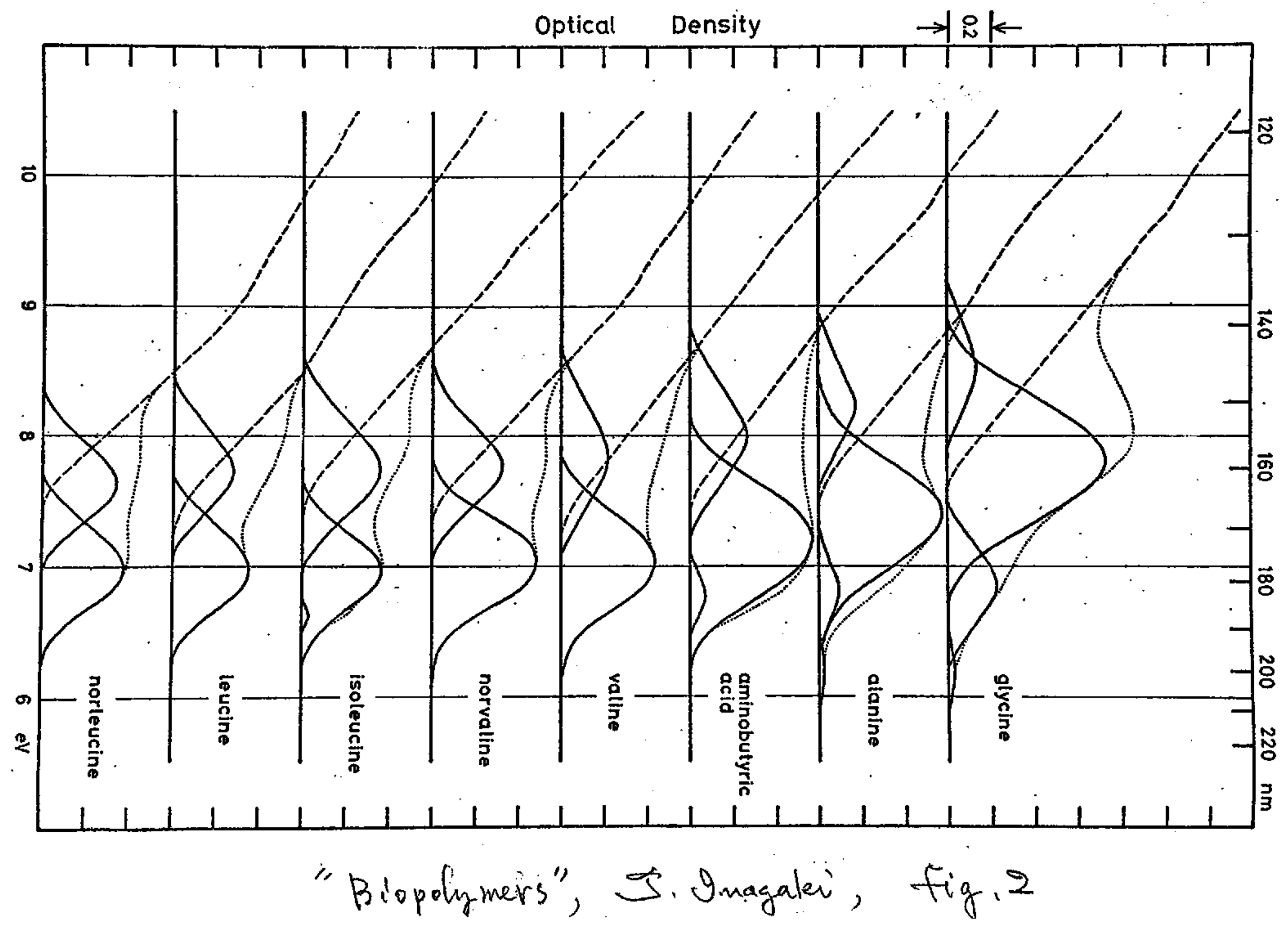




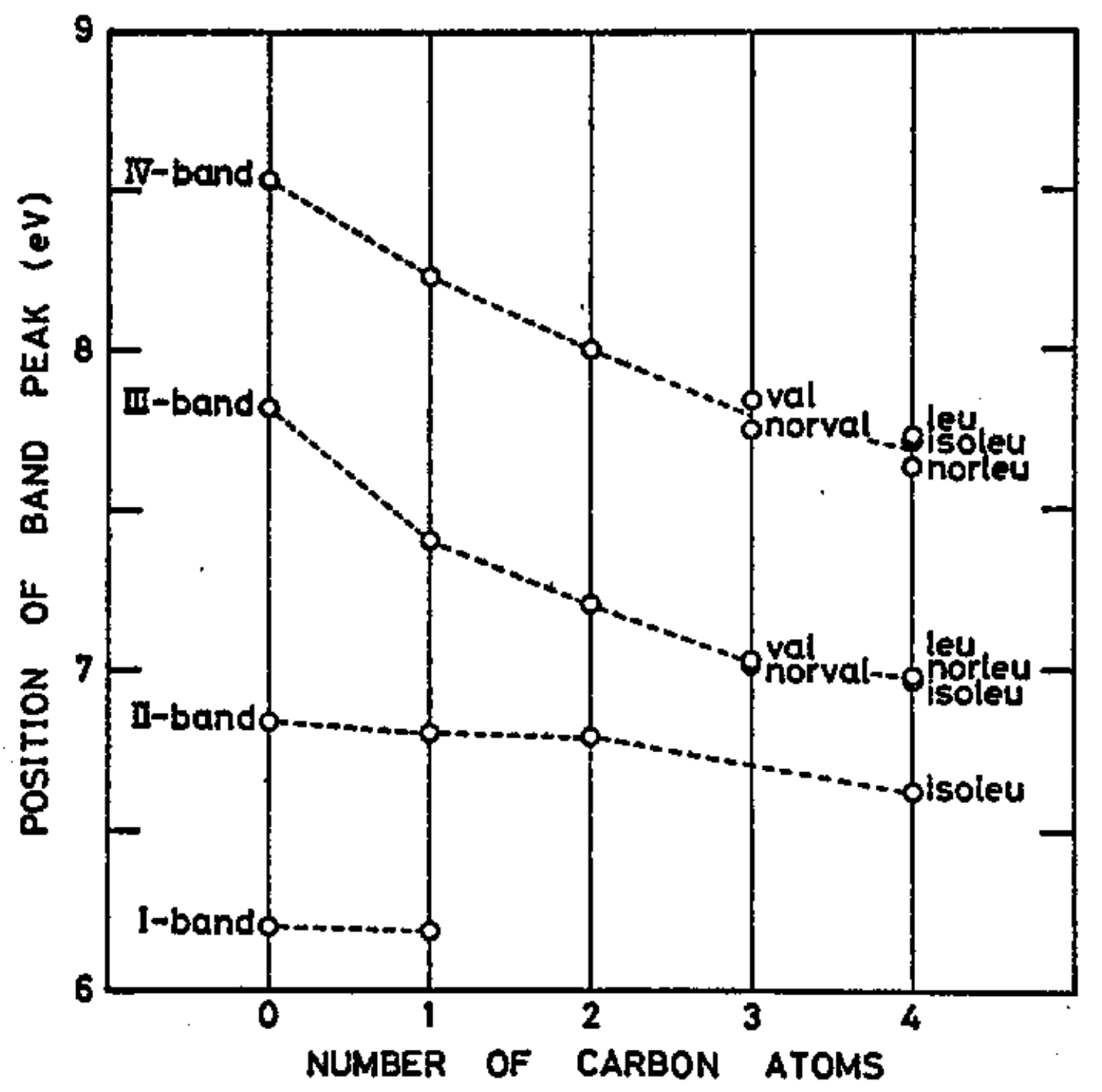

"Biopolymers", I. Inagaki, fig. 3 